Citius, Altius, Fortius- 2019, 12(2), pp. 3-13

\title{
LOS VALORES OLÍMPICOS EN LA REFORMA DE LA EDUCACION ESCOLAR PROPUESTA POR PIERRE DE COUBERTIN (1889-1937): UNA EDUCACION PARA LA PAZ, LA LIBERTAD Y LA DEMOCRACIA ${ }^{1}$
}

\author{
M. ${ }^{\text {a }}$ Eugenia Martínez-Gorroño \\ Universidad Autónoma de Madrid (Spain) \\ eugenia.martinez@uam.es \\ Fecha de recepción: julio 2019 \\ Fecha de aceptación: octubre 2019
}

DOI: http://doi.org/10.15366/citius2019.12.2

Resumen:

El presente trabajo es resultado de la investigación histórica realizada en torno a las relaciones que el Olimpismo, la ideología Olímpica y el Movimiento Olímpico moderno han mantenido en torno a la paz. Pierre de Coubertin propuso una reforma de la educación que fomentaba en las escuelas valores como la paz, la libertad y la democracia. Proporcionó respuestas de orientación claramente pacifistas a los problemas de la sociedad de su tiempo y a la dirección beligerante que se estaba confiriendo a la Educación Física escolar. Coubertin estaba motivado por un temor al creciente resurgimiento del nacionalismo, el impulso al vigor de las «razas», el papel patriótico que se quería imponer a los hombres como significado del deber de la masculinidad, así como la manipulación política de esos aspectos. En su reforma educativa Coubertin trataba de ofrecer alternativas para una educación liberadora, pacífica y democrática, que diera respuesta a las necesidades de las personas, no a los intereses de los poderes fácticos de aquellos momentos.

Palabras clave: Paz, Reforma de la educación, Pierre de Coubertin, Olimpismo, Movimiento Olímpico, valores, deporte.

Title: OLYMPIC VALUES IN THE SCHOOL EDUCATION REFORM PROPOSED BY PIERRE DE COUBERTIN (1889-1937): EDUCATION FOR PEACE, FREEDOM AND DEMOCRACY

\begin{abstract}
:
The present work is the result of historical research carried out around the relations that the Olympism, Olympic ideology and the modern Olympic Movement have maintained around peace. Pierre de Coubertin proposed a reform of education which encouraged values like peace, freedom and democracy in schools. He provided clearly pacifist guidance answers to the problems of society in his time and the bellicose direction of physical education; it was motivated by his fear of the growing reemergence of nationalism, the impact of the "races" power, the patriotic role that they wanted to impose on men such as the meaning of the duty of masculinity, as well as the manipulation of all its aspects. These were alternatives for a liberating, peaceful and democratic education, which responded to the needs of individuals as persons and not to the interests of the factual powers of these years.
\end{abstract}

Keywords: Peace, Educational reform, Pierre de Coubertin, Olympism, The Olympic Values, sport.

\footnotetext{
${ }^{1}$ El presente trabajo es la versión en español de la conferencia que fue presentada en francés por la autora en la $12^{a}$ Sesión Internacional para Directores y Presidentes de Academias Olímpicas celebrada en la Academia Olímpica Internacional en su sede de Olimpia (Grecia) en mayo del 2014.
} 


\section{Deporte en las Escuelas: La Orientación Educativa de Pierre de Fredy}

El Olimpismo moderno partió de la inquietud social de Pierre de Coubertin (1863-1937), preocupado por los problemas de la sociedad de su tiempo, quien creyó poder enfrentarlos con éxito a partir de educar a la sociedad utilizando como instrumento el deporte, siempre que éste fuera practicado siguiendo el ideario que elaboró como base del Movimiento Olímpico.

El estudio de su trayectoria nos permite identificar dos objetivos diferentes en su idea de educar: por un lado, la educación de la sociedad en su conjunto, con la creación del Movimiento Olímpico y los Juegos Olímpicos, éstos últimos como el evento cumbre del primero. Por otro lado, la educación de las personas en edad de formación, tratando de cambiar el contenido que se impartía en las escuelas.

Este trabajo se centra en el segundo de los impulsos, la reforma educativa que pretendía llevar a cabo en los colegios utilizando el deporte como recurso educativo. Esta idea de cambiar los métodos de enseñanza estuvo presente desde sus primeras inquietudes. Hay referencias escritas de este proyecto desde 1887; aunque su primera puesta en práctica se inició en 1889 en el Colegio Monge de París. Este proyecto por reformar los métodos educativos escolares le llevó a emprender esfuerzos diferentes a aquellos que realizó para impulsar la celebración de los Juegos Olímpicos modernos.

Los primeros impulsos para difundir primero la necesidad y luego la idea de esta reforma de los métodos educativos fueron realizados en mayo de 1888 con la fundación del Comité para la propagación de los Ejercicios Físicos en la Educación. Con aquella fundación inició su Campaña de «Rebronzer la France» con la que pretendía reformar la Educación francesa e introducir los juegos y los deportes como formas de Educación Física. Con ese motivo escribía en una carta a los miembros de la Sociedad de Economía Social, pidiéndoles su apoyo:

«Hay que hacer la reforma social a través de la educación. No hay que trabajar con los adultos, sino con los niños... poner el ejercicio físico como contrapeso a la fatiga intelectual... intentando que en la educación entren, mediante el deporte, nuevos principios de disciplina y de responsabilidad»².

En algunos otros momentos, a partir de las fuentes de sus escritos, podemos encontrar claramente como el propio Coubertin diferenció esta reforma de los métodos de enseñanza, del otro objetivo que era el restablecimiento la celebración de los Juegos Olímpicos. Por ejemplo, en una carta que escribió en 1924 a su amigo Franz Reichel, precisamente tras la edición de los Juegos de París de aquel mismo año, cuando abandonó la Presidencia del Comité Olímpico Internacional. En ella afirmaba:

«... jamás me desentenderé del Olimpismo y de los deportes, pero mis sesenta y cinco años están luchando con una labor nueva que reclama todo su esfuerzo: la transformación de las bases de los métodos de enseñanza» (Le Figaro, Vol.73, 8 de julio, 1927, p.3)

\section{El punto de partida}

La práctica de ejercicios físicos en su forma competitiva agonística y en su función educativa (Hernández Álvarez, J.L., 1996,1997), eran potencialidades que ya habían sido utilizadas para instruir

\footnotetext{
${ }^{2}$ Coubertin, P. (1889) Lettre aux members de la Société d'Économie Sociale et des Unions de la Paix Sociale, en La Réforme sociale ( $1^{\circ}$ septembre 1888$)$ vol. 8, série, 2 part. VI, pp. 249-252. Reimpresa en L'Éducation Anglaise en France, Paris, Libr. Hachette, 1889, pp. 199-202, Ap. II.

Citius, Altius, Fortius Volumen 12 ne 2. Noviembre, 2019. ISNN. 2340-9886.

http://doi.org/10.15366/eitius2019.12.2
} 
y educar a los jóvenes por varias culturas de la antigüedad. Para Coubertin aquella función fue un redescubrimiento personal a partir de analizar la forma de educar anglosajona y tras unos viajes cuyo objetivo fue conocer los sistemas educativos de varios países. Como es sabido, posteriormente, emprendió una lucha vital para convencer de su utilidad e introducir la práctica deportiva como recurso educativo en la Europa Continental.

Los biógrafos de Pierre de Coubertin afirman que estudió Ciencias Políticas en la Universidad de la Sorbona, por lo que su preparación universitaria no estuvo orientada hacia la enseñanza. Sin embargo, su inquietud social y su vocación filantrópica, le llevó a afirmar:

«He decidido cambiar bruscamente mi carrera en el deseo de unir mi nombre al de una gran reforma pedagógica» ${ }^{3}$. (Pierre de Coubertin)

$\mathrm{Y}$ en muchos otros momentos, aquella inquietud social por poner solución a los problemas de la sociedad en la que se desenvolvía le llevó a expresar su confianza en que sólo la educación podía poner remedio a aquella problemática:

«En el estado actual del mundo, de Europa en particular, ninguna reforma de orden político, económico o social podrá ser fecunda sin una reforma previa de la pedagogía» ${ }^{4}$.

Los autores que han escrito sobre aquella reforma escolar que impulsó Coubertin, han apuntado que estaba motivada porque en las enseñanzas que se impartían en los centros docentes de la Europa continental primaban los contenidos «más intelectuales», y porque veía abandonada la educación de los aspectos corporales. Sin contradecir estas afirmaciones, pensamos además que las circunstancias sociales y políticas en las que se desenvolvía Pierre de Coubertin tuvieron una influencia decisiva en la orientación de aquella reforma, circunstancias que no han sido nunca mencionadas y que nosotros vamos a analizar en los siguientes apartados.

\section{Las circunstancias sociopolíticas de la Europa de Pierre de Coubertin en la base de su propuesta de una educación para la paz}

Los avatares de aquella Europa convulsa en la que se desenvolvió Coubertin, pensamos que condicionaron en forma definitiva la orientación que quiso dar a la reforma educativa que pretendía introducir en los colegios.

Revisando varios de sus escritos, podemos recoger sus menciones y sus denuncias sobre las orientaciones que los sectores de poder, sin ningún criterio moral, pretendían dar a la educación, manipulando las ignorancias y promoviendo las injusticias, difundiendo en los centros docentes las orientaciones nacionalistas que convenían a sus intereses y que contradecían los derechos de los ciudadanos. Todo ello, sin duda, motivó y estuvo presente en la connotación pacífica que conformó la reforma de la enseñanza que sobre los contenidos escolares pretendía Coubertin y que pasamos a analizar.

La Educación Física que en aquellos años se impartía en los centros docentes de toda Europa continental se centraba en la gimnasia. Su contenido de ejercicios analíticos, rígidos y autoritarios pretendían formar principalmente a los alumnos varones, con unos objetivos encaminados al endurecimiento de su cuerpo y de su carácter. Aquel adiestramiento disciplinante estaba muy próximo

\footnotetext{
${ }^{3}$ Vialar, P. (1962). Pierre de Coubertin, The Man, The Games. The International Olympic Academy, p. 16.

${ }^{4}$ Coubertin, P. (1933?) Bulletin BIP, no 5 p. 3-5. Cita p.3.
} 
a la instrucción militar. Con todo ello, gran número de estados y poderes fácticos de aquellos momentos intentaban y conseguían manipular las tendencias agonísticas y competitivas que en los aspectos de las capacidades físicas surgen de forma natural entre los varones y, que, durante los años de adolescencia y juventud, les sirven para sus necesidades de autoafirmación con frecuencia pretendidamente masculina.

Aquel tipo de Educación Física respondía a la orientación, criterios y tendencias que imperaban entre los gobiernos europeos de aquel periodo. Así, en la Francia de Pierre de Coubertin surgió por ejemplo la Ligue de L'Éducation Physique, dirigida por Pascal Grousset, que defendía que los alumnos varones deberían ser iniciados en el adiestramiento físico con orientación militar desde sus primeros años, y que ese debía ser el contenido de la Educación Física que debía ser impartido en los centros escolares. Aquellas tendencias de educación belicista se vieron acentuadas por la derrota francesa frente a los alemanes en 1870. Los intereses del gobierno y de varios sectores sociales pretendían aprovechar la etapa educativa para preparar y fomentar el espíritu de revancha entre la población, a fin de recuperar los territorios perdidos de la Alsacia y la Lorena. Estas circunstancias también impulsaron y pusieron de moda el surgimiento de lo que fue conocido como los Bataillons Scolaires en los que nos detendremos posteriormente.

Aquel ambiente «militarizante» de los contenidos educativos, fue alcanzando vigencia en la Francia en la que vivía Coubertin y, por ejemplo, algunos senadores republicanos franceses como E. Georges y B. Saint-Hilaire defendían que la preparación militar debía comenzar en la escuela, en cuyos recintos la gimnasia y el tiro deberían ser impartidos obligatoriamente a los alumnos varones.

Pero el problema fue más importante cuando aquellas tendencias belicistas se fueron materializando en los contenidos educativos siendo reguladas oficialmente. Así, en Francia, siendo Jules Ferry Ministro de Enseñanza Pública y Bellas Artes, se publicaron varias leyes entre 1880 y 1882 estableciendo los contenidos de la Enseñanza Primaria. Así, una Ley de 28 de marzo de 1882 sobre la Enseñanza Primaria Obligatoria estableció: «La enseñanza primaria comprende...La gimnasia;... para los muchachos los ejercicios militares».

La Gimnasia y el tiro ${ }^{6}$ debían ser impartidos como contenidos obligatorios de la Educación Física de los varones de los centros de enseñanza dependientes del Estado, de los Departamentos y de los Municipios.

Esto supuso que la escuela se convertía en el primer centro de pre-instrucción militar. Pierre Arnaud reflexionando sobre la trayectoria, la orientación y los precedentes de aquella ley escribía:

«La Ley de 1880, principalmente dictada por motivos militares y políticos, trataba de hacer de las escuelas la antecámara del cuartel a fin de preparar la revancha. La ley completaba desde aquella perspectiva las disposiciones de 1868 y 1872 y se justificaba igualmente por una nueva concepción de

\footnotetext{
${ }^{5}$ Loi de 28 mars 1882 sur l'enseignement primaire obligatoire. Journal Officiel de la République Française, pp. 1-2. Textualmente en francés : "L'enseignement primaire comprend : ... La gymnastique ; ... pour les garçons les exercices militaires ${ }^{5}$.

${ }^{6} \mathrm{Si}$ bien el tiro empezó a formar parte de los contenidos escolares, fue regulado por Decretos posteriores, como el del 27 de julio de 1893 firmado por el Ministro R. Poncaré, que estableció la creación de una comisión encargada de controlar la enseñanza del tiro en las escuelas y a la que se encargó elaborar un reglamento de tiro y la elección del arma más apropiada para usar en las escuelas.
}

Citius, Altius, Fortius Volumen 12 ne 2. Noviembre, 2019. I§NN. 2340-9886.

http://doi.org/10.15366/eitius2019.12.2 
la educación general que había sido esbozada desde 1868 por Victor Duruy, Jules Simón y Octave Gréard»?.

Pierre de Coubertin se manifestó específicamente en contra del contenido de aquellas leyes:

«El régimen (de educación) actual engendra agotamiento físico, con frecuencia agotamiento intelectual y siempre debilidad moral.... el proyecto de militarizar la educación y que los ejercicios militares sirvan de contrapeso... lograrán unas mentes todavía menos abiertas y unos caracteres cada vez más anodinos; tenemos ya bastantes borregos en nuestro país: que no se nos den todavía más, cosa que desde luego ocurriría confundiendo dos disciplinas que apenas se parecen: la disciplina militar y la disciplina escolar, acercando a dos seres que no se parecen en absoluto: el soldado y el niño» ${ }^{8}$.

Pero las tendencias belicistas siguieron materializándose. Los profesores que debían impartían las clases de Educación Física que establecieron las leyes de 1880 y 1882 reforzaron aún más las orientaciones militaristas de la Educación Física que se impartía en los colegios de Francia. $\mathrm{Al}$ no haber docentes preparados para impartir los nuevos contenidos, fueron sectores cercanos al ejército los encargados generalmente de su impartición. Este aspecto coadyuvó especialmente a que las maneras, enfoques y orientaciones militares fueran tomando cada vez más presencia y cercanía en los ámbitos de los niños y jóvenes en periodo formativo.

«Son militares o antiguos militares voluntarios quienes aseguran las clases de gimnasia, de tiro y de instrucción militar dentro de los colegios de primaria, los institutos y los liceos». «Los programas, tanto como los que los aplican, contribuyen a hacer de la gimnástica una instrucción cripto-militar dominada por la disciplina y los ejercicios de orden. La instauración del tiro en 1881 y más tarde de los batallones escolares en 1882 no hicieron más que acentuar esta tendencia»?.

Las tendencias belicistas continuaron imponiéndose a partir de otras disposiciones legales. El Decreto del 6 julio de 1882 firmado por Jules Grévy y Jules Ferry instituyó legalmente los Batallones Escolares. Se trataba de una organización que como forma de actividad física extraescolar completaba las enseñanzas gimnásticas y las perspectivas de instrucción militar para los niños de edades comprendidas entre los 10 y los 12 años. Los Batallones Escolares tuvieron un gran impulso y alcanzaron gran vigencia.

Cada batallón debía estar compuesto por 300 alumnos, escogidos entres los escolares de la comunidad. Sobre el objetivo explícito e implícito de la creación de los mismos afirmaba Pierre Arnaud: «Era el símbolo del patriotismo de la juventud, de su voluntad de reconquistar las provincias perdidas en $1870{ }^{10}$.

Empezaron a constituirse en diferentes lugares Batallones Escolares como testimonio vivo de la fidelidad republicana de las comunidades. Por ejemplo, Lyon, muy pronto contó con dos Batallones Escolares gracias al apoyo de su alcalde el doctor Gailleton y del Consejo Municipal que dedicaron un presupuesto importante al equipamiento de los alumnos: uniforme y fusil.

\footnotetext{
${ }^{7}$ Arnaud, P. (1985) Les lois sur 1’Enseignement de la gymnastique en La Mémoire du Sport. Centre de Recherche et d'innovation sur le sport. U.E.R.E.P.S. Université Lyon 1, p. 30-31, citation p.30.

${ }^{8}$ Coubertin, P. (1889). Le remède au surmenage, en L'Éducation Anglaise en France, Paris, Hachette, pp-3-20.

${ }^{9}$ Arnaud, P. (1985) Op. Cit. p. 30.

${ }^{10}$ Arnaud, P. (1985). Les débuts du mouvement sportif dans la région lyonnaise, en La Mémoire du Sport. Centre de Recherche et d'innovation sur le sport. U.E.R.E.P.S. Université Lyon 1. p. 32.
} 
«Los pequeños soldados de la República no dejarán de desfilar, el día de la fiesta nacional (instituida en 1880) bajo los ojos de una loca admiración, y en presencia de los más notables militares y políticos» ${ }^{11}$.

Sobre aquellos hechos y circunstancias, pueden encontrarse en los escritos de Coubertin, múltiples denuncias y respuestas. Como alternativa a aquella Educación Física, rígida, disciplinante y especialmente sometedora, Pierre de Fredy propuso introducir el deporte en los centros escolares de enseñanza, imitando las formas inglesas. El deporte era visto por Coubertin como recurso educativo modernizante, liberador, democrático, motivador y pacifista, que podría dar, a la Educación Física que se impartía, una muy diferente orientación como materia escolar en la Europa continental de la segunda mitad del siglo XIX.

Muchas veces denunció aquellas manipulaciones desde los ámbitos del poder, que veía patentes y que orientaban la enseñanza de los colegios.

«La idea de la preparación militar activada y perfeccionada por el ejercicio físico es muy antigua. En Caldea, como en Egipto y en Extremo Oriente, los gobiernos belicistas y conquistadores recurrieron a este sistema y es probable que hicieran un llamamiento al instinto deportivo, para cooperar con sus deseos» ${ }^{12}$.

Especialmente, después de la Primera Guerra Mundial, podemos ver como resaltó las consecuencias de la estimulación de la preparación física, que, manipulada por los estados con ambiciones de poder, debían ser denunciadas y neutralizadas. En la siguiente cita textual podemos observar claramente los temores de Coubertin ante la problemática nacionalista y belicista de la Europa en la que se desenvolvía:

«Antes de que la Guerra Mundial se desatara, ya sabíamos que el renacimiento deportivo, por el cultivo de las energías individuales, había creado fuerzas nacionales, y la gran tragedia de hoy lo ha probado de modo perentorio y sangriento. Pero el deporte puede hacer algo más por nosotros. Nos asegurará, para el mañana, si sabemos permitírselo, el bien esencial, fuera del cual no es posible ningún arreglo permanente: la paz social» ${ }^{13}$.

Al igual que Pierre de Coubertin, diferentes sectores democráticos estaban firmemente preocupados por la circunstancia de que, en todos los países europeos, importantes grupos sociales se manifestaban especialmente temerosos de que fuerzas agresivas foráneas a sus naciones pudieran aprovechar la supuesta «debilidad física» o falta de potencial agresivo militar, para invadir y adueñarse de sus territorios. Con aquella perspectiva o justificación se esforzaban por convencer y preparar a los jóvenes varones para que estuvieran concienciados, preparados y siempre dispuestos para defender a sus respectivas patrias, idea a la que igualmente iba unido el odio al extranjero, identificado como enemigo. Todo ello se planteaba unido a la propaganda de mostrarlo como un deber básico que los verdaderos hombres adultos debían cumplir para demostrar que lo eran.

Los gobiernos europeos de las últimas décadas del siglo XIX impulsaban similares orientaciones educativas similares. Importantes sectores de toda Europa veían en la Educación Física

\footnotetext{
${ }^{11}$ Ibidem

${ }^{12}$ Coubertin, P. (1918). Lo que podemos pedir ahora al deporte. (Comunicación hecha en la Asociación de Helenos Liberales de Lausanne el 24 de febrero de 1918). Citius, Altius, Fortius. Humanismo, sociedad y deporte: Investigaciones y Ensayos, Vol. $2 n^{\circ}$ 2. Madrid, Comité Olímpico Español-CEO de la Universidad Autónoma de Madrid. 2009, pp. 135-144. Cita p. 125 .

13 Ibídem, p. 135.

Citius, Altius, Fortius Volumen 12 ne 2. Noviembre, 2019. ISNN. 2340-9886.

http://doi.org/10.15366/eitius2019.12.2
} 
una materia escolar ideal para inculcar a los niños varones un pretendido patriotismo, bajo el cual se escondían sus intereses para formar ejércitos para cumplir sus ambiciones y deseos de ampliar su poder y sus territorios. Se pretendía pues, a partir de aquellos contenidos de la Educación Física, inculcar los roles y los deberes que debían ser asumidos por los hombres como benefactores de su patria, y se denunciaban como objetos de olvido que debían ser impulsados y cultivados.

A partir del análisis de los escritos de Coubertin, puede verse como, bajo aquel ambiente, el parecía vislumbrar y ver concretamente las circunstancias que estuvieron en el origen de la Segunda Guerra Mundial. Se evidencia en sus palabras los temores y los anuncios preclaros sobre el germen de nuevas guerras:

«Los tiempos que se avecinan son graves para la humanidad. Los apetitos plutocráticos insaciables y la sed de dominio llevada hasta la locura, por un lado, y, por otro, el levantamiento contra las injusticias, largo tiempo soportadas, contribuyen a mantener la civilización bajo amenazas de posguerra, que podría ser peor que la guerra misma» ${ }^{14}$.

También en el Bulletin du Bureau International de Pédagogie Sportive. que empezó a publicarse en la sede del Comité Olímpico Internacional en Lausana en 1928, y que se presentó como el órgano cultural y educativo del Movimiento Olímpico ${ }^{15}$, Coubertin denunció la utilización y el acercamiento hacia el deporte de ciertos gobiernos que pretendían impulsarlo, no con objetivos educativos, sino por sus pretensiones belicistas y de poder. Y así escribía:

«En nuestros días parece que ciertos gobiernos que se ocupan ardientemente del organismo deportivo lo hacen para satisfacer sus instintos nacionalistas más que por el mejoramiento simple de la salud pública, así como también existen grupos deportivos internacionalizados que se inspiran en una preocupación étnica o en una preocupación de clase» ${ }^{16}$.

\section{Las propuestas de Pierre de Fredy para la Educación Física Escolar a fin de impulsar valores educativos como la solidaridad, la cooperación, la convivencia universal, la paz y la democracia}

Como ya hemos dicho, la reforma de los métodos educativos de los centros escolares estuvo en los primeros proyectos de Pierre de Coubertin. En mayo de 1888 fundó el Comité pour la propagation des Exercices Physiques dans l'Éducation. En 1889 impulsó la creación de la Asociación para la Reforma de la Educación Física en Francia y se puso al frente como Secretario General, convocando el 20 de mayo de aquel mismo año el Congreso para la Propagación de los Ejercicios Físicos en la Educación.

Coubertin, con sus inquietudes por buscar la paz y fomentar la libertad insistió en mostrar la etapa escolar como un periodo de la vida que debía permitir experimentar la sensación de libertad y que debía potenciar el vigor físico sin reprimir los impulsos corporales. En el colegio se debía educar y orientar a los niños; pero nunca manipularlos para conseguir que se pusieran al servicio de las ambiciones políticas y los deseos de poder y dominio de los gobiernos belicistas. Así escribía:

\footnotetext{
${ }^{14}$ Ibídem, p. 144.

${ }^{15}$ Krüger, A. (2011): «Citius -Altius - Fortius? Armonía física o superación sin límites en las últimas obras de Pierre de Coubertin según el Bulletin du Bureau International de Pédagogie Sportive» en Citius, Altius, Fortius. Humanismo, sociedad y deporte: Investigaciones y Ensayos, Vol. 4 n 2 , pp.119-135.

${ }^{16}$ Pierre de Coubertin, Bulletin BIP n ${ }^{\circ} 5$ p. 3-5. ¿1933? Cita p. 3.
} 
«La educación debe ser, yo lo repito, el prefacio de la vida. El hombre será libre, el niño debe serlo también. Se trata de enseñarle solamente a usar su libertad y a comprender su importancia [...] Libertad para el cuerpo, libertad para el espíritu, libertad para el carácter, libertad para todo» ${ }^{17}$.

«Las restricciones son a veces útiles, pero la libertad debe estar en la base de todas las instituciones escolares» ${ }^{18}$.

Frente a aquellas normas legislativas citadas en los apartados anteriores, la reforma escolar que Pierre de Coubertin propuso, ofrecía varias respuestas concretas ante aquellas disposiciones. Como ya hemos mencionado, frente a la gimnasia con ejercicios analíticos, rígidos y disciplinantes, proponía los juegos y el deporte como contenido de las clases de Educación Física, con una orientación liberadora, solidaria y de cooperación, como instrumento para la educación humanista. Era el rol que él indicaba que desempeñaban los juegos y los deportes en la educación inglesa. «Un rol que es, a la vez, físico, moral y social» ${ }^{19}$.

Como alternativa a los exmilitares como profesores de Educación Física, en 1889 Coubertin, entonces a cargo de la Secretaría General de la Asociación para la Reforma de la Educación Escolar en Francia, propuso un nuevo tipo de profesorado de Educación Física, que debía estar formado por voluntarios libres y laicos, ajenos a los principios marcados por la administración o por servidumbres de tipo ideológico o religioso.

Como actividad fuera del horario escolar, frente a los Batallones Escolares, propuso «la formación de pequeños clubes deportivos para educar a los niños en el autogobierno», según la inspiración Arnoldiana de las Escuelas Públicas Británicas, persiguiendo el objetivo de educar desde la competición pacífica. Recordemos el siguiente párrafo, ya citado:

«El deporte puede hacer algo más por nosotros. Nos asegurará para el mañana, si sabemos permitírselo, el bien esencial más allá del cual no es posible un acuerdo permanente: la paz social» ${ }^{20}$.

La reforma social para una convivencia pacífica a partir de la educación fue también un propósito que estaba en la base de los impulsos de Pierre de Coubertin y que sentía como imprescindible para una sociedad que en el siglo XIX debía orientar sus miras hacia la democracia.

«¿Cómo, entonces, podemos preparar a las masas trabajadoras para que desempeñen su papel democrático? Ciertamente no tomando el camino constitucional o político, sino el camino educativo, la fórmula que podría reemplazar viejas injusticias sin apoyarse en nuevas injusticias» ${ }^{21}$.

Con respecto a que la reforma educativa que impulsaba tuviera una orientación de base democrática podemos encontrar muchas citas entre los escritos de Coubertin. Por ejemplo, en 1918 escribía: «El Olimpismo...se niega a admitir la existencia de una enseñanza de lujo, reservada a las clases acomodadas que no sería accesible a las clases trabajadoras». Coubertin siempre denunció

\footnotetext{
${ }^{17}$ Coubertin, P. (1887). L'éducation anglaise. Comunicación presentada en la Société d'économie social el 18 abril de 1887. Publicada en Les Études Sociales. No 137, 2003, 1er. Semestre. Ed. Société d’Économie et de science sociales. París, pp.125144. Cita p. 130.

${ }^{18}$ Coubertin, P. (1890) Conclusions en Universités Transatlantique, Paris. Lib Hachette, 1890, pp.361-379.

${ }^{19}$ Ibidem, p. 135.

${ }^{20}$ Coubertin, P. (1918) "Lo que podemos pedir ahora al deporte". Op. Cit .p. 135.

${ }^{21}$ Coubertin P. en Clastres, P. (2003)b. La décennie leplaysienne de Pierre de Coubertin. De la réforme des Lycées aux Jeux olympiques (1883-1896). Les Études Sociales, 137, 1er semestre, 5-19. Revue publiée par la Société d'économie et de science sociales. París, pp. 5-19. Cita p.12.
} 
las desigualdades sociales. Siempre le parecieron el origen de las revoluciones. La falta de oportunidades educativas eran una injusticia que pensaba debía ser superada, indicando la necesidad de igualdad de oportunidades para todos los sectores sociales:

«La educación [la inglesa] es libre, como corresponde a una nación emancipada; no produce el desmantelamiento, la plaga de nuestro país que es la causa muchas revoluciones» ${ }^{22}$.

Por ello, muchos fueron los análisis que hizo de la necesidad de educar a todos los sectores sociales y muchos sus argumentos sobre la necesidad de educar para crear una sociedad democrática. Argumentos que utilizó, por ejemplo, para promover también otras iniciativas como la creación de una universidad obrera. «...ya sea que en un próximo futuro la clase obrera ejerza el poder, ya sea que simplemente participe en él, no por ello la cuestión de su preparación es menos primordial» ${ }^{23}$.

Así, Coubertin argumentó hasta la saciedad en múltiples conferencias y escritos, la necesidad de una reforma de los contenidos y métodos de la educación para que los niños y los jóvenes estuvieran preparados para la democracia. Para ello, el deporte ofrecía unas características definitivas para aprender a cooperar pacíficamente:

«En la sociedad democrática la asociación es el origen de toda fuerza útil y la base de todo esfuerzo lógico»... «cualesquiera que sean los gobiernos de una democracia, sus instituciones, sus aspiraciones... ésta necesita la cooperación para vivir y prosperar... esta cooperación necesita ser aprendida y se lleva mucho ganado si se aprende desde la juventud. Esta verdad se impondrá en la democracia, la cual se verá obligada a introducir la cooperación en la vida escolar para preparar a los futuros ciudadanos con vistas a la vida activa» ${ }^{24}$.

La necesidad de construir entre todos una sociedad democrática en la que todos participasen era el motivo por el que estaba convencido de los beneficios de una educación orientada a potenciar y valorar el esfuerzo, para la que el deporte era un elemento educativo y un recurso idóneo y excelente.

«El ciudadano más útil para la democracia será... el que entre en la vida activa entrenado ya en el esfuerzo colectivo, acostumbrado inconscientemente a los movimientos, al ritmo y a la contención que este esfuerzo exige; y esta persona no quiere tener una formación teórica, sino sólo práctica» ${ }^{25}$.

«De la educación transformada esperamos ciudadanos que no necesiten recurrir a semejantes procedimientos (revoluciones); ciudadanos activos y determinados que tengan una divisa ...que amen a Dios, a la patria y a la libertad $»^{26}$.

En resumen, podemos afirmar que Coubertin, como señaló Clastres, mantuvo:

«Un ciclo de sesenta años de lucha contra las escuelas secundarias dirigidas como cuarteles, oponiéndose a los colegios con internados militarizantes y contra el jacobinismo pedagógico ${ }^{27}$.

\footnotetext{
${ }^{22}$ Coubertin, P. (1888) L'Éducation en Angleterre. Collèges et Universités. Paris, Libr. Hachette, p. 1-23.

${ }^{23}$ Coubertin, P. (1922) Entre deux batailles. De L’Olympisme à L’Université ouvriere, en La Revue de la Semaine, 3e. année, 20 janvier. 1922 n 1 pp. 299-310.

${ }^{24}$ Coubertin, P. (1901) Notes sur l'Éducation Publique, cap. XIII. París, Lib. Hachette. Pp. 217-235.

${ }^{25}$ Ibídem.

${ }^{26}$ Coubertin, P. (1889) Le remède au surmenage en L'Éducation anglaise en France, París. Lib. Hachette, pp. 3-20.

${ }^{27}$ Clastres, P. (2003a). L'éducation des jeunes élites: Un nouveau chantier de la paix sociale? Les Études Sociales, 137, 1er semestre. Revue publiée par la Société d'économie et de science sociales. 145-153 París. Citación p. 145.
} 
Por ello, propuso una reforma de la educación que impulsara dentro de los colegios valores como la paz, la libertad y la democracia.

Coubertin dio respuestas claramente pacifistas a los problemas de la sociedad de su tiempo y a la orientación belicista de la Educación Física; motivado por los temores que le suscitaba el creciente resurgir de los nacionalismos, el impulso de la orientación de la idea de las «razas», el supuesto papel patriótico que se pretendía imponer como deber de masculinidad, y la manipulación política de todos estos aspectos. Como alternativas, proponía una educación liberadora, pacífica y democrática que respondiera a las necesidades de las personas como individuos y no a los intereses de los poderes fácticos de aquellos años.

\section{BIBLIOGRAFÍA Y FUENTES}

\subsection{Archivos}

— Archivo del Comité Olímpico Internacional. Lausana (Suiza)

- Archivo del Comité Olímpico Español. Madrid (España)

- Archivo de la Academia Olímpica Española. Madrid (España)

- Archivo de la Biblioteca Nacional de España. Madrid (España)

- Archivo de la Biblioteca Virtual del Patrimonio Bibliográfico

\subsection{Bibliografía utilizada como fuente}

— IOC. (2018). Charte Olympique. Lausanne: International Olympic Committee.

- Coubertin, P. (1887). L'éducation anglaise. Comunication faite à la Société d'économie social. Séance du 18 avril 1887. Les Études Sociales, 137, 2003, ler. Semestre. París: Société d’Économie et de science sociales.

- Coubertin, P. (1933?). España en la cultura física y los Deportes. Bulletin BIP, 5, 3-5. (Texto publicado por primera vez en España en Citius, Altius, Fortius. Humanismo, sociedad y Deporte: Investigaciones y ensayos,4(8), 132).

- Coubertin, P. (2009). Lo que podemos pedir ahora al deporte (Conferencia pronunciada en la Asociación de Helenos Liberales de Lausana el 24 de febrero de 1918). Citius, Altius, Fortius. Humanismo, sociedad y deporte: Investigaciones y Ensayos, 2(2), 135-144. Comité Olímpico Español- CEO de la Universidad Autónoma de Madrid.

— Coubertin, P. (1973). Ideario Olímpico. Discursos y Ensayos. INEF. Madrid.

- Coubertin, P. (2011). Olimpismo. Selección de Textos. Comité Internacional Pierre de Coubertin. Lausana. 


\subsection{Bibliografía y referencias bibliográficas}

- Arnaud, P. (1985). Les Lois sur 1’Enseignement de la gymnastique. En Mémoire du sport. Ed. Centre de Recherche et d'Innovation sur le sport. U.E.R.E.P.S. Université Lyon 1.

- Clastres, P. (2003)a. L'éducation des jeunes élites: Un nouveau chantier de la paix sociale? Les Études Sociales, 137, 1er semestre, 145-153. Revue publiée par la Société d'économie et de sciencie sociales. París.

- Clastres, P. (2003)b. La décennie leplaysienne de Pierre de Coubertin. De la réforme des Lycées aux Jeux olympiques (1883-1896). Les Études Sociales, 137, 1er semestre, 5-19. Revue publiée par la Société d’économie et de sciencie sociales. París.

- Clastres, P. (2010). La aceptación de la República por Pierre de Coubertin. Discurso inédito pronunciado ante la Conferencia Molé de Paris en 1887. Citius, Altius, Fortius. Humanismo, Sociedad y Deporte: Investigaciones y Ensayos, 3(2), 39-59. Comité Olímpico Español-Centro de Estudios Olímpicos de la UAM. Madrid.

- Krüger, A. (2011). Citius -Altius - Fortius? Armonía física o superación sin límites en las últimas obras de Pierre de Coubertin según el Bulletin du Bureau International de Pédagogie Sportive. Citius, Altius, Fortius. Humanismo, sociedad y deporte: Investigaciones y Ensayos,4(2), 119135. Ed. Comité Olímpico Español-CEO de la UAM. Madrid.

- Martínez Gorroño, M.E. (2014). Les valeurs olympiques dans la réforme de l'éducation scolaire proposée par Pierre de Coubertin (1889-1937): une éducation pour la paix, la liberté et la démocratie. En 12th Joint International Session for Presidents or Directors of National Olympic Academies and Officials of National Olympic Committees. Ancient Olympia (Greece): International Olympic Academy.

- Martínez Gorroño, M.E. (2008). Los festivales panhelénicos y los Juegos Olímpicos: puntualizaciones, análisis y revisiones históricas. Citius, Altius, Fortius. Humanismo, sociedad y deporte: investigaciones y ensayos, 1(1), 25-51. Comité Olímpico Español-CEO de la Universidad Autónoma de Madrid. ISSN 188-6744.

- Martínez Gorroño, M.E. (2012). El Olimpismo y la paz. En M.E. Martínez Gorroño y C. Durántez Corral (Coords.), Movimiento Olímpico: Historia y rectos actuales, (pp. 29-54). Madrid: Servicio de Publicaciones de la Universidad Autónoma de Madrid.

- Solar Cubillas, L.V. (2003). Pierre de Coubertin. La dimensión pedagógica. La aportación del Movimiento Olímpico a las pedagogías cor

- Tamayo Fajardo, J.A. (2003). Superación de los conflictos de orden internacional por parte del Movimiento Olímpico de la era moderna a lo largo del siglo XX. Efdeportes. Revista Digital, 9(59). Buenos Aires (Argentina) 